\title{
Experiments about Roughness of Wood Composites
}

\author{
Aurel LUNGULEASA \\ Transilvania University of Brasov, Romania, lunga@unitbv.ro \\ Cosmin SPIRCHEZ \\ Transilvania University of Brasov, Romania, cosmin.spirchez@unitbv.ro
}

\begin{abstract}
The paper presents some values of roughness for the main composite wooden materials. A device Mitutoyo with diamond tip for determining the roughness was used, fixed on a vertical device taken from Kennon Instruments. The values of roughness obtained for the main analyzed wooden composites were compared with massive wood, after that they were correlated with the 14 classes of quality provided by the European standard in the field EN ISO 4287:1997. The obtained results make possible a better knowledge of the wooden composites presently used in the wooden industry, from the point of view of surface smoothness. A general rule for increasing or decreasing the roughness according to the type of composite wooden material was not noticed surely, all roughness depending on the state and surface material (finished or not, veneered or not calibrated or not).
\end{abstract}

\section{Keywords}

roughness, composites, absolute deviations, average square root, average maximum height

\section{Introduction}

The beauty of the wood is a consequence of its complex structure, and a smooth surface highlights this beauty. The smoothness of a wooden surface is relative and depends along the processing operations and the size of the sectioned anatomical elements (wooden porosity) which appear on that surface. That is why it may say that while processing wood we will not get a perfect smoothness. The quality of the surface of a wooden material is quantified by the roughness, which determines the size of the asperities given by the processing operation and the wood anatomy. The roughness of a wooden surface is defined as assembly of the micro-irregularities or the average deviations, which form the relief of the surface and defines finally its quality. Taking into consideration that the roughness depends on the processing manner [1], as well as on the size of the anatomic elements of the wood, the roughness of the wooden surface presents as holes, porosity, superficial fissures, striations, grooves and sometimes even fibers and chip tearing [2]. When processing wooden surfaces there will appear three types of irregularities, respectively macro unevenness and waves [3], these all being on the same surface, or only individually. The roughness will depend on all these types of unevenness. If for the solid wood the surface smoothness depends on the porosity of the wood species and is precarious, for the composite materials (which have a more uniform structure than the solid wood) the quality of the surface is more uniform and more predictable [4, 5].

The methodology used to determine the surfaces roughness [6] base on certain values, on 2D feeling of the surfaces using a stylus technique usually with a diamond tip and on the 3D optical method (with the luminous section method or the shadowed section). Stylus technique has a lot of disadvantages (such as contact with wood of stylus will scratch the surface, head size makes possible the missing of fine irregularities, slow moving of head, etc.) but it is still used in the techniques of assessing the wood roughness [7]. There are a multitude of parameters of expression of the surface roughness of all the materials and especially for wood, but among them more important are considered the following: Rathe arithmetic mean of the absolute deviations, Rq- average square mean, and Rz- the average maximum height. These parameters are defined and expressed in EN ISO 4287 (1997) [8], EN ISO 4288 (1996) [9] and DIN 4768 (1990) [10].

The arithmetic mean of absolute deviations. This parameter is indicated by Ra and represents the arithmetic mean of the absolute deviations of the roughness profile in comparison to the average line. For this it is necessary to know the line of the roughness profile and a series of values for each of the heights and the depths of this profile. Based on their values the arithmetic mean is obtained, respectively 
their center line. In comparison to the central line there are obtained several deviations, some of them situated on the upper side of the central line and other under it (Figure 1).
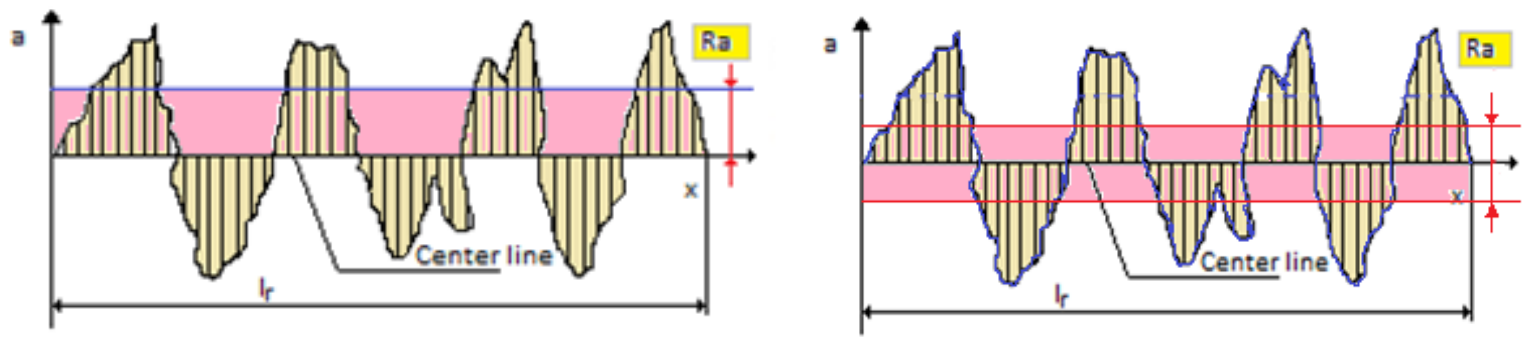

Fig. 1. Determining the Ra parameters (a-based on the relation 1, b-based on the relation 2) a - deviation; $l_{r}$ - length of reference

If there are considered the ones above the line as being positive and the ones under the line as being negative there is a zero line, which is the central line. In the moment the deviations under the central line, they are considered as positive, and the ones above the central line are still positive and then the arithmetical mean of roughness is found (Eq. 1)

$$
R a=\frac{\sum_{i=1}^{n} a_{i}}{n}
$$

were $a_{i}$ represents absolutely value of medium deviation from average for roughness profile, in $\mu \mathrm{m}$, and $n$ - number of measured deviations.

If it is taken into consideration that the deviations under the central line of the profile are negative, their module will be used or the positive absolute values in the previous relation (Eq. 2) become as follows:

$$
R a=\frac{\sum_{i=1}^{n} a_{s i}+\sum_{j=1}^{m}\left|a_{i j}\right|}{n+m}
$$

where there are: $a_{s i}$ - current superior deviation, in $\mu \mathrm{m} ; a_{i j}$ - inferior current deviation, in $\mu \mathrm{m}, n$ - number of superior deviations; $m$ - number of inferior deviations.

Average square mean Rq. The Rq parameter represents the average square mean of parameter Ra, and it is a more sensitive parameter than Ra, being used in optical applications. In order to obtain this parameter, the values of $\mathrm{Ra}$ are firstly calculated, after which the 2-grade radical is extracted from it (Figure 2).

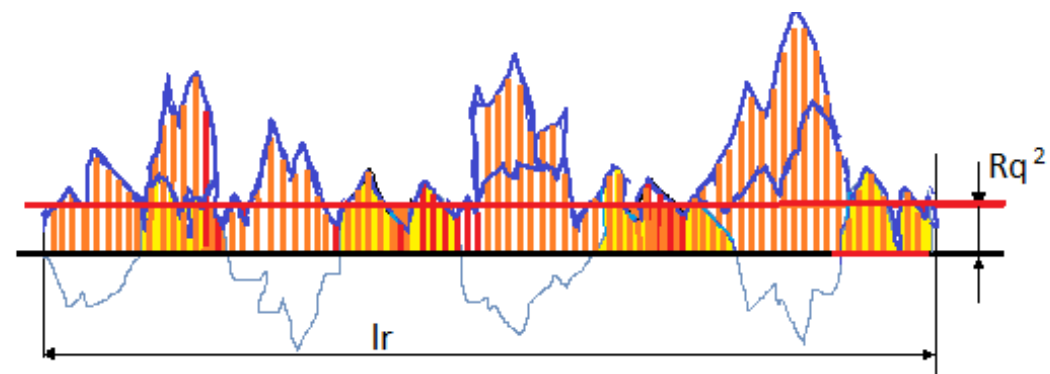

Fig 2. Determining the Rq parameter of roughness

The average maximum height $\mathrm{Rz}$. In order to determine the Rz parameter, the roughness profile is determined, and then five areas of reference are considered, where the highest top and the deepest depth are found for the profile. The height between them is noted with $R z_{1}, R_{2}, \ldots, R z_{5}$ (Figure 3). The arithmetic mean is calculated for the two heights and the value of parameter $\mathrm{Rz}$ is found. 


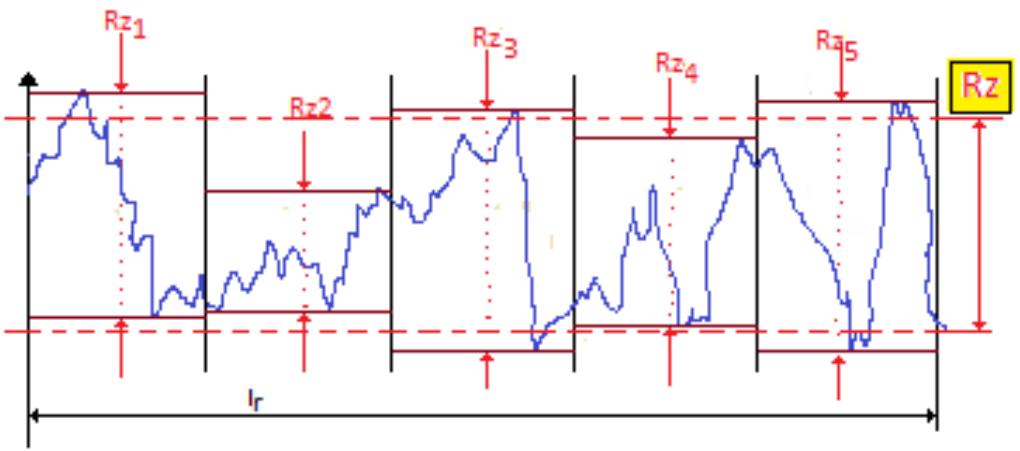

Fig 3. Determining the Rz parameter

The standard ISO 8402: 1986 found a correlation between the two main parameters of roughness: $\log (\mathrm{Rz})=0.65+0.97 \times \log (\mathrm{Ra})$. Standard EN ISO 4287: 1997 [8] indicates that there are 14 classes of roughness marked from N0 to N13, where Ra parameter varies from 0.012 to $100 \mu \mathrm{m}$ (Table 1 ).

Table 1. Correlation between classes of roughness and Ra parameter (ISO 4287:1997)

\begin{tabular}{|c|c|c|c|c|c|c|c|c|c|c|c|c|c|}
\hline N0 & N1 & N2 & N3 & N4 & N5 & N6 & N7 & N8 & N9 & N10 & N11 & N12 & N13 \\
\hline 0.012 & 0.025 & 0.05 & 0.10 & 0.20 & 0.40 & 0.80 & 1.60 & 3.20 & 6.30 & 12.50 & 25.00 & 50 & 100 \\
\hline
\end{tabular}

If the values in Table 1 are introduced in an Excel chart (Figure 4), it is noted that the exponential function is the one which characterizes more the value tendency, with a perfect correlation coefficient Pearson $\mathrm{R}^{2}=1$.

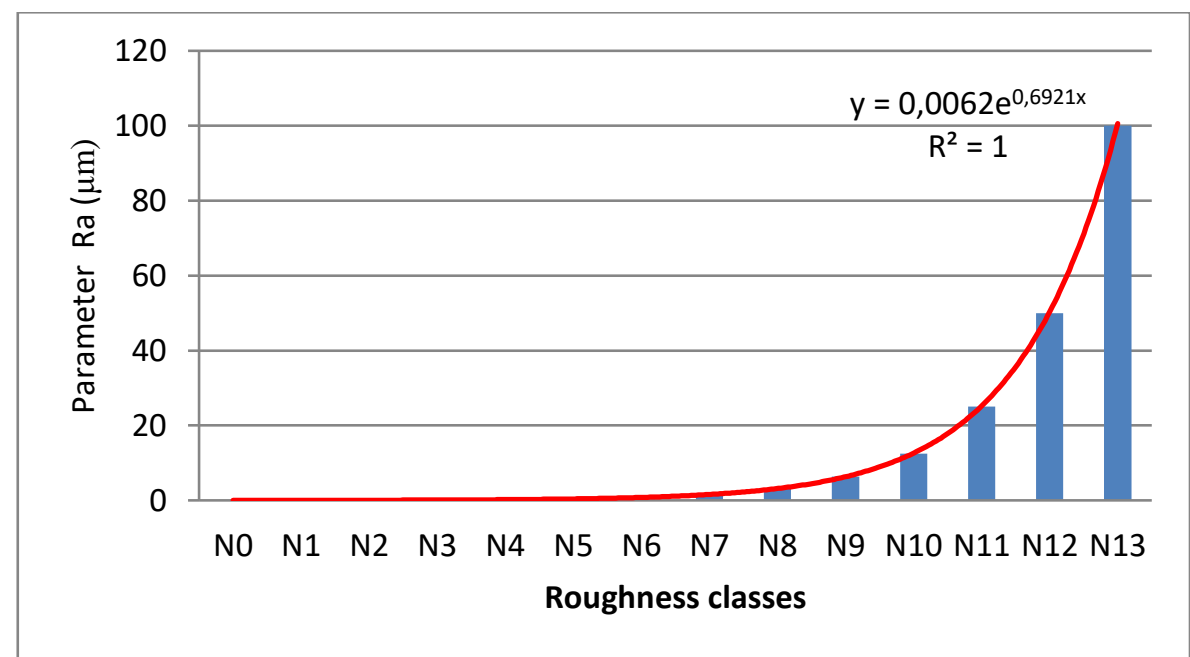

Fig. 4. The Correlation between the roughness classes and the values of parameter Ra

The main object of the paper is to obtain a roughness of the surface of different wooden composites, expressed by the three parameters, $\mathrm{Ra}, \mathrm{Rq}$ and $\mathrm{Rz}$, helping by a roughness device with diamond feeler. Also, it is wanted to make the correlation of the roughness of these materials with roughness classes. This thing is necessary for a better compatibility between the composites surface and other covering materials such as: veneer, raisins, coating paper, melamine foils, etc.

\section{Materials and Installation}

The device to determine the roughness of wooden materials consisted in a Mitutoyo type inductive phertometer SJ-201 (with diamond tip feeler and a Gaussian digital filter), which have functions according to EN ISO 4287: 1997 [8], fixed on a Kennon Instruments columns support (Figure 5). The wooden piece 1 subject to the action to determine the roughness is disposed on a perfectly horizontal 
support, with the working head 2 above it. The working head, which is fixed elastically on a joint in order to overtake the unevenness of the piece, is adjusted in two directions and they are:

- regarding the vertical direction, the diamond tip is raised or lowered 1-2 $\mathrm{mm}$ with the hand wheel 3, such that the working head is in direct contact with the wooden piece whose roughness is determined. It will be taken into consideration that the device allows a vertical movement of maximum $6 \mathrm{~mm}$;

- regarding the horizontal direction adjustment, the diamond tip is positioned at about 3-4 $\mathrm{mm}$ on the table end, known the fact that when feeling the surface, the diamond tip has a measurement movement of about $4 \mathrm{~mm}$ backwards to the test sample end, afterwards it moves ahead in order to return to the initial position with a speed of $0.5 \mathrm{~mm} / \mathrm{s}$ on the same distance of $4 \mathrm{~mm}$.

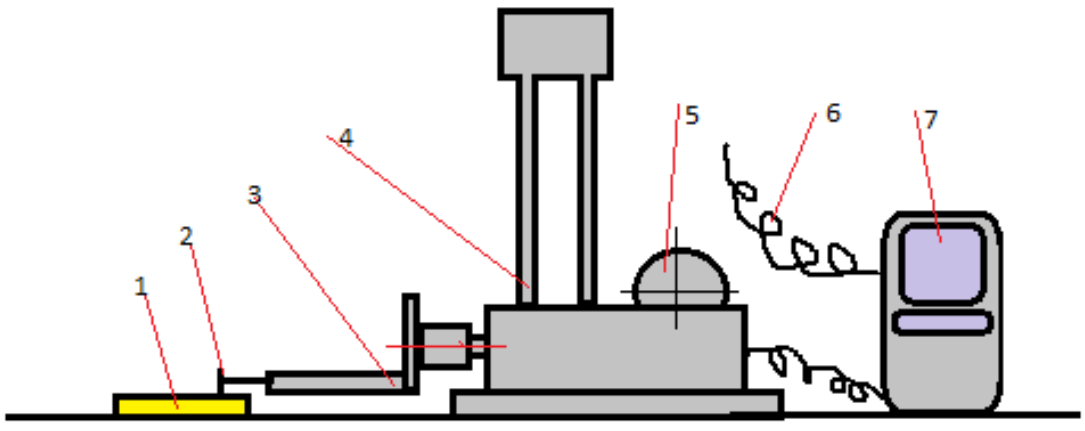

Fig. 5. Mitutoyo SJ-201 phertometer

1-wooden piece; 2-working head with diamond tip; 4-two columns support; 5-hand wheel for vertical movement; 6-supply cable; 6-phertometer screen

The working head is fixed on a massive support with two columns 4, for an appropriate lifting of the diamond tip for pieces with large and different thickness. The device is composed of a supply cable 5 at $230 \mathrm{~V}$, a transformer at $12 \mathrm{~V}$, a massive support 6 in cast iron and the display screen of the three parameters of roughness $\mathrm{Ra}, \mathrm{Rz}$ and $\mathrm{Rq}[11]$.

The used composite material was transformed in test samples of $50 \times 50 \mathrm{~mm}$ and consisted of chipboards, classical fiberboards, medium -density fiberboard (MDF), wooden - plastic composites (WPCs), etc. (as it is indicated in Figure 6).

For comparison it was used beech wood, smoothed or polished with granulation 100 . These boards were marked as follows:

- Chipboard 1 is a calibrated chipboard;

- Chipboard 2 is an enameled chipboard;

- Chipboard 3 is an un-calibrated chipboard (gross);

- Plywood 1 is a calibrated interior plywood;

- Plywood 2 is exterior plywood whose faces were covered with a phenol film.

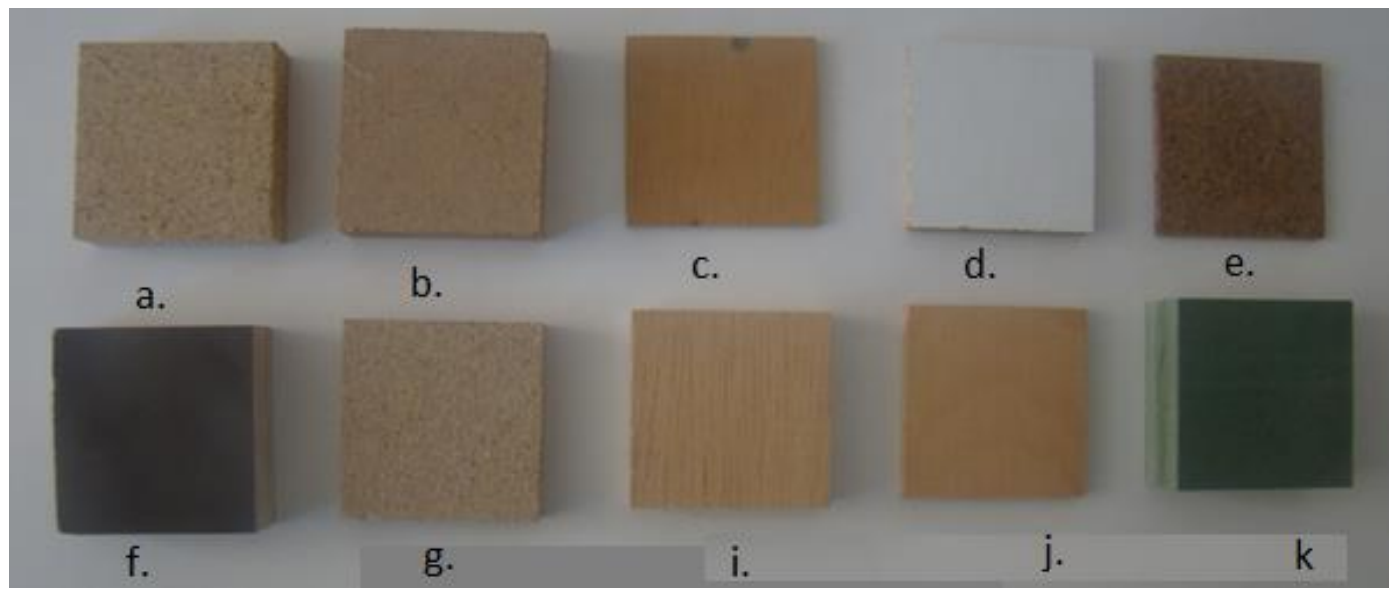

Fig. 6. Types of used composite materials 


\section{Results and Discussion}

After determining the three parameters of roughness $\mathrm{Ra}, \mathrm{Rq}$ and $\mathrm{Rz}$, a comparison between different wooden composite materials could be performed. A first noticeable aspect was that the condition of the surface and its roughness do not depend on the composite board density. In this manner we have similar parameters of roughness $(\mathrm{Ra}=6.2 \mu \mathrm{m}, \mathrm{Rq}=7.8 \mu \mathrm{m}$ and $\mathrm{Rz}=35.1 \mu \mathrm{m})$ for panel and massive wood (although the massive wood density of $690 \mathrm{~kg} / \mathrm{m}^{3}$ is a lot higher than the panels of $430 \mathrm{~kg} / \mathrm{m}^{3}$. The explanation in this case depends on the fact that the panel has at the surface applied an esthetic veneer whose structure is similar to the one of the massive woods. There are significant differences between calibrated and non-calibrated chipboard, to which even if there are close densities $\left(740 \mathrm{~kg} / \mathrm{m}^{3}\right)$, the roughness parameters values are different, being for calibrated chipboard smaller $(\mathrm{Ra}=6.09 \mu \mathrm{m}$, $\mathrm{Rq}=8.20 \mu \mathrm{m}$ and $\mathrm{Rz}=39.62 \mu \mathrm{m})$ than the non-calibrated one $(\mathrm{Ra}=12.96 \mu \mathrm{m}, \mathrm{Rq}=15.97 \mu \mathrm{m}$ and $\mathrm{Rz}=66.21 \mu \mathrm{m})$. The explanation for this difference is that the calibrated chipboard is smoother than the non-polished one, the roughness of the gross chipboard being given by the size of the surface particles and the recovery grade after pressing.

There is a superior quality of some surfaces of wooden composites in comparison to the massive wood, when it is desired a finishing of these products during the pressing process. Thus, the lowest values of roughness are for WPCs, melamine chipboard and filmed plywood, (respectively a Ra under $3.2 \mu \mathrm{m}$ ), and the highest values are for gross boards (gross chipboard with Ra under $50 \mu \mathrm{m}$ ) or the calibrated ones (calibrated plywood, calibrated MDF and veneered plywood with Ra under $50 \mu \mathrm{m}$ ). All these values may be notices in Figure 7 and Figure 8.

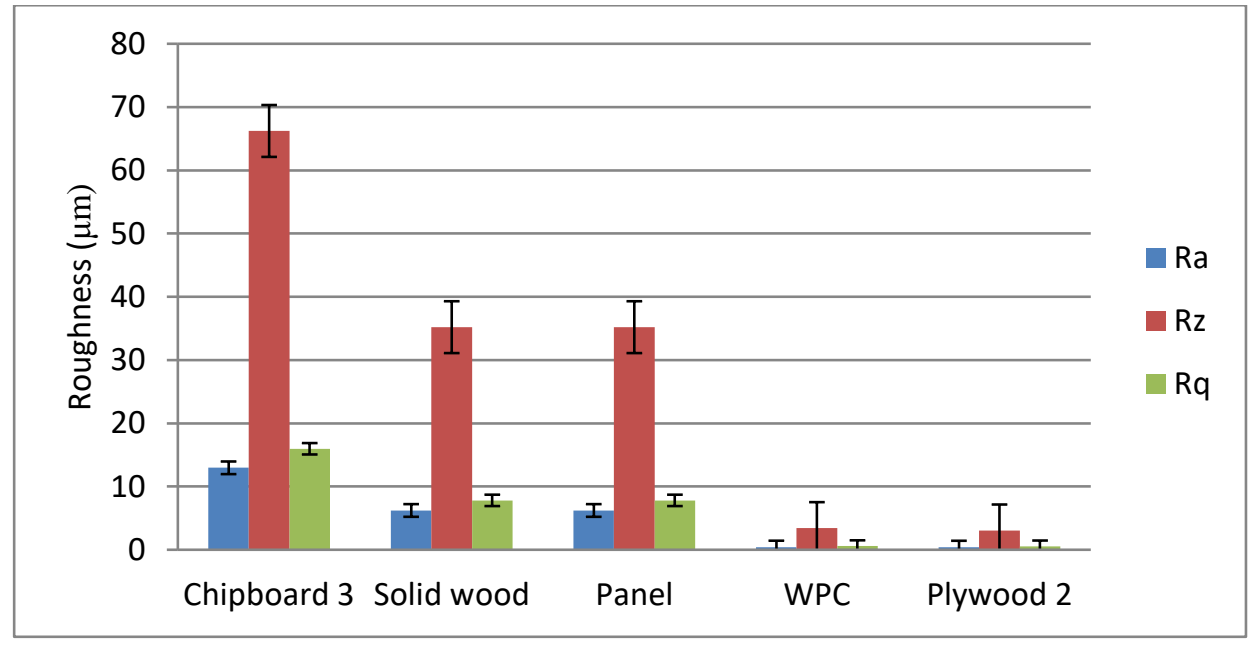

Fig. 7. Roughness parameters for some wood composites

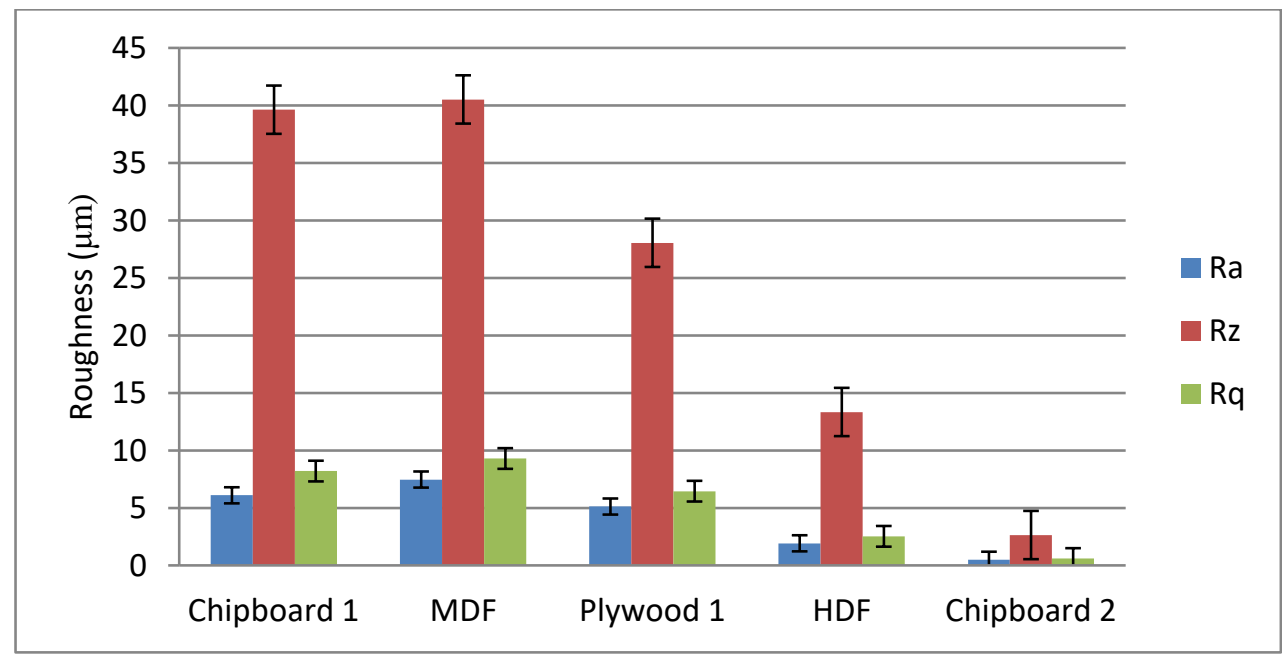

Fig 8. Roughness parameters for some wood composites 
Comparatively with the solid wood the veneered boards are similar as roughness, the gross boards have a higher roughness, and the finished ones a lower roughness. Thus, as a general conclusion, the composite materials subject to roughness analysis, may have a higher roughness (than the massive wood) if they are in gross or calibrated condition or a lower roughness if they are finished by applying a phenol film or a melamine foil [12].

According to the values of roughness Ra parameter and the standard EN ISO 4287:1997 [8] the analyzed composites may be classified in quality classes (Table 2).

Table 2. The correlation between the roughness classes and the Ra parameter, superiorly limitative values

\begin{tabular}{|c|l|c|c|c|c|c|}
\hline Class & \multicolumn{1}{|c|}{ N8 } & N9 & N10 & N11 & N12 & N13 \\
\hline Ra, $\mu \mathrm{m}$ & \multicolumn{1}{|c|}{3.20} & 6.30 & 12.50 & 25.00 & \multicolumn{1}{c|}{50} & 100 \\
\hline Board type & $\begin{array}{l}\text { WPC, } \\
\text { Filmed play-wood, } \\
\text { Melamine Chipboard }\end{array}$ & -- & --- & HDF & $\begin{array}{l}\text { Solid wood, } \\
\text { Sanded chipboard, } \\
\text { Veneered panel, } \\
\text { MDF, Plywood }\end{array}$ & $\begin{array}{l}\text { Brut } \\
\text { chipboard }\end{array}$ \\
\hline
\end{tabular}

It is noted that all the composite boards are classified over 8 class of roughness, here being WPC, filmed Play-wood and melamine Chipboard. Then it is HDF (wet process) in class N11, then solid wood, sanded chipboard, veneered panel, MDF and plywood in class N12 and gross chipboard in class N13.

\section{Conclusion}

The roughness of composite materials is important especially when these are used for furniture and other decorative objects, by veneering. The roughness offers itself an esthetic aspect, and when veneering is made, it is determined the adhesive quantity which is used. Following the experiments conducted, in the paper was noticed that the different composite materials have a different roughness. There is no general rule for the increasing or decreasing of composite wooden materials roughness, their roughness depending on the condition of processing and the material that is put on the composite surface.

\section{References}

1. Kilica M., Hiziroglu S., Burdurlu E. (2006): Effect of machining on surface roughness of wood. Building and Environment, ISSN 0360-1323, Vol. 41, issue 8, p. 1074-1078, https://doi.org/10.1016/i.buildenv.2005.05.008

2. Thoma H., Peri L., Lato E. (2015): Evaluation of wood surface roughness depending on species characteristics. Maderas. Ciencia y tecnología, ISSN 0718-221X, Vol. 17, no. 2, p. 285-292, http://dx.doi.org/10.4067/S0718221X2015005000027

3. Magoss E. (2008): General Regularities of Wood Surface Roughness. Acta Silv. Lign. Hung., Vol. 4, p. 81-93

4. Hiziroglu S., Jarusombuti S., Fueangvivat V. (2004): Surface characteristics of wood composites manufactured in Thailand. Building and Environment, ISSN 0360-1323, Vol. 39, issue 11, p. 1359-1364, doi.org/10.1016/ j.buildenv. 2004.02.004

5. Ulker O. (2018): Surface Roughness of Composite Panels as a Quality Control Tool. Materials, ISSN 1996-1944, Vol. 11, 407, doi:10.3390/ma11030407

6. Gurau L., Irle M. (2017): Surface Roughness Evaluation Methods for Wood Products: a Review. Current Forestry Reports, Vol. 3, p. 119-131, https://doi.org/10.1007/s40725-017-0053-4

7. Hiziroglu S. (1996): Surface roughness analysis of wood composites: A stylus method. Forest Products Journal 46(7), p. 67-72

8. EN ISO 4287: 1997. Geometrical product specifications (GPS). Surface texture. Profile method. Terms. Definitions and surface texture parameters. International Organization for Standardization

9. EN ISO 4288: 1996. Geometrical product specifications (GPS) - Surface texture. Profile method. Rules and procedures for the assessment of surface texture. International Organization for Standardization

10. DIN 4768: 1990. Determination of Surface Roughness Values of the Parameters by Means of Electrical Contact (stylus) Instruments; Terminology, Measuring Conditions. Deutsches Institut für Normung

11. Mitutoyo (1984): Quick guide to surface roughness measurement. Reference guide for laboratory and workshop. Bulletin No. 2229, Mitutoyo America Corporation

12. Zhong Z.W., Hiziroglu S., Chan C.T.M. (2013): Measurement of the surface roughness of wood based materials used in furniture manufacture. Measurement, ISSN 0263-2241, Vol. 46, issue 4, p. 1482-1487, https://doi.org/10.1016/j.measurement.2012.11.041 